\title{
Analysis and computation of non-equilibrium two-phase flows
}

\author{
ABHIJIT GUHA \\ Department of Aerospace Engineering, University of Bristol, Queen's Building, \\ University Walk, Bristol BS8 1TR, UK \\ e-mail: A.Guha@Bristol.ac.uk
}

\begin{abstract}
Non-equilibrium fluid mechanics and thermodynamics of twophase vapour-droplet and gas-particle flow are considered. Formation of the droplets as well as their subsequent interaction with the vapour are discussed. A new theory of nucleation in steam turbines is developed that reproduces many aspects of measured droplet size spectra which cannot be explained by any available steady-flow theories. (Steam turbines are responsible for $80 \%$ of global electricity production and the presence of moisture significantly reduces turbine efficiency costing 50 million pounds per annum in UK alone.) Fluid dynamic interactions discussed include flow instabilities induced by condensation, condensation wave theory, relaxation gas dynamics for vapour-droplet flow, thermal choking due to non-equilibrium condensation, the structure of shock waves and their development through unsteady processes, and jump conditions and the interpretation of total pressure in two-phase flows.
\end{abstract}

Keywords. Non-equilibrium fluid mechanics; thermodynamics of two-phase flows; nucleation in steam turbines.

\section{Introduction}

Two-phase flow of a vapour-liquid mixture consisting of a large number of minute liquid droplets uniformly dispersed throughout a background vapour phase continuum is both scientifically interesting and of considerable engineering importance (in a variety of areas in mechanical, chemical and aerospace engineering, and meteorology). In the present paper, we discuss the formation of the droplets as well as their impact on the subsequent thermo-fluid dynamics of the flow. We restrict ourselves mainly to the description of pure substances, i.e., when both phases are of the same chemically pure species. Numerical illustrations are given only for steam-water mixtures, but the general principles are applicable to other substances as well.

What follows is a brief description, with no equations, of some of the work with which the present author has been involved over the past few years. The references cited (Guha 
\& Young 1989, 1991, 1994; Young \& Guha 1991; Guha 1992, 1994, 1997) give a fuller treatment of these topics. The VKI lecture series (Guha 1995) treats most of these topics at much greater depth. It also contains a good repertoire of references and works of many researchers in this field which we do not reproduce here owing to space constraints.

\section{Thermo-fluid dynamics of condensation}

\subsection{Physical description of homogeneous condensation}

All condensing (or evaporating) flows are non-equilibrium to a greater or less extent. Departures from equilibrium are measured by the subcooling $\Delta T$ which is the difference between the saturation temperature at local pressure and the actual vapour temperature $\left(\Delta T=T_{s}-T_{g}\right) . \Delta T$ governs the rate at which nuclei are formed as well as the rate at which established droplets grow (or evaporate).

As pure, clean steam expands through a nozzle or a turbine blade passage, droplets do not appear as soon as the condition line crosses the saturation line. This is due to the existence of a free-energy barrier involved in creating new surface area. For some considerable time during expansion the steam remains dry in a metastable equilibrium until the subcooling becomes high enough to trigger an appreciable nucleation rate. Depending on the rate of expansion and the pressure, steam may become subcooled by $30-40^{\circ} \mathrm{C}$ while still remaining dry. The nucleation process leads to the formation of very large number $\left(10^{14}-10^{17}\right.$ nuclei per $\mathrm{kg}$ of steam) of tiny droplets (diameter $<1 \mathrm{~nm}$ ), called the primary fog, more or less uniformly distributed in the continuous vapour phase. Nucleation is practically terminated at the point of maximum subcooling called the Wilson Point. For pure steam, if the Wilson points for tests with varying nozzle inlet conditions are plotted on the equilibrium Mollier diagram, they are contained within a narrow zone around a line called the Wilson line (which corresponds to approximately $3-4 \%$ equilibrium wetness line).

The droplets thus formed then rapidly grow in size by exchanging heat and mass with the surrounding, subcooled vapour (the final droplet radii, $r$, in laboratory nozzles lie in the range $0.02-0.2 \mu \mathrm{m}$ ). The high rate of heat release as a result of rapid condensation, causes a sharp increase in vapour temperature and consequently an exponential decay of the subcooling. Depending on the values of the flow parameters, the initial growth phase of the droplets may give rise to a gradual increase in pressure known as "condensation shock". The term "shock", however, is a misnomer. Although pressure rises as a result of heat addition to supersonic flow, the Mach number downstream of the condensation zone usually remains above unity and, more importantly, the rise in pressure is gradual.

In conventional laboratory nozzle experiments, where (dry saturated or superheated) steam is produced in a boiler, the flow must expand to supersonic velocities for significant subcooling to develop. However, if subcooled steam could be supplied at the nozzle inlet, homogeneous condensation could occur in the subsonic part of the flow. This is possible, for example, in a multistage turbine where steam could become subcooled at the inlet of a blade row as a result of work extraction in previous blade rows. Subsonic condensation would result in a decrease, rather than an increase, in pressure. 
Some inlet conditions may give rise to combinations of flow Mach number and heat release rate that necessitate the formation of a true aerodynamic shock wave inside the condensation zone ("supercritical condensation shock"). Under certain conditions this shock wave may become unstable and propagate towards the nozzle throat. The compressive wave ultimately interferes with the nucleation zone causing a reduction in nucleation rate and hence heat release rate. With the cause of its inception removed, the strength of the wave decreases and the flow again expands through the throat in a shock-free manner thus allowing the whole process to repeat itself. Such unsteady flow is normally observed in pure steam when the inlet stagnation temperature $T_{0}$ is close to the saturation temperature at the inlet stagnation pressure $p_{0}$. Homogeneous condensation then occurs in the transonic region close to the throat causing flow instability (the flow domain and boundary conditions remaining fixed).

Keeping $p_{0}$ fixed, if $T_{0}$ is progressively reduced from superheated to subcooled levels, one encounters different regimes of homogeneous condensation in the order: Subcritical condensation (the usual pressure humps characteristic of many condensation experiments), supercritical condensation (with inbuilt shock wave), oscillatory condensation and subsonic condensation (Guha 1994a).

After the "condensation shock", the steam generally reverts to near thermodynamic equilibrium at which the temperature of the vapour as well as of the droplets is close to the saturation level. Since the growth of liquid phase takes place by heat transfer through a finite temperature difference between the phases, the process is essentially irreversible and has associated with it a net rise in entropy. In turbines this appears as a reduction in the potential for performing work and is referred to as the thermodynamic wetness loss. This is a major component of the overall wetness loss. A simplistic version of an empirical rule, formulated by Baumann in 1921, states that the efficiency of a steam turbine decreases by $1 \%$ for every $1 \%$ increase in mean wetness fraction. A typical value of the wetness fraction at the exit of a steam turbine in an electricity-generating power-plant is $10-12 \%$. Thus, in the last stages, the wetness loss is comparable to the combined effects of the profile, secondary and tip leakage losses. A 1988 estimate by the then Central Electricity Generating Board of UK showed that the adverse wet steam effects cost them 50 million pounds per year. The global implication is thus quite serious, since steam turbines are responsible for about $80 \%$ of the world-wide generation of electricity and there is a considerable economic incentive for further research.

\subsection{Numerical solutions for different regimes of condensation}

The numerical scheme for the calculation of steady as well as unsteady non-equilibrium wet steam flow has been detailed in Guha \& Young (1991) and Guha (1994a). Here, we describe only the outline and highlight some important aspects.

One of the most effective methods of calculation is to write a computational "black-box" which contains the nucleation and droplet growth equations, and the energy equation in its thermodynamic form. Together they furnish the full set of equations that describe completely the formation and growth of liquid droplets in a fluid particle (from a Lagrangian viewpoint), if the pressure-time variation is specified. The pressure-time variation is obtained by time-marching solutions of the conservation equations such as Denton's method, 
extensively used for single-phase calculations in turbomachinery blade rows. In this respect, the thermodynamic aspects of phase-change can be completely divorced from fluid dynamical considerations so that the use of the "black-box" is effectively independent of any particular computational fluid dynamic application. Thus established single-phase CFD codes can, rather easily, be modified to deal with non-equilibrium two-phase flow with the above-mentioned modular approach. (The flexibility of this scheme may be appreciated from Guha \& Young (1994) where the same "black-box" has been grafted into a streamline curvature calculation procedure.)

The development of the computational routines within the "black-box" represents a comparatively major undertaking and has been fully described by Guha \& Young (1991) and Guha (1994a). The routines are sufficiently general and robust to deal with any type of nucleating or wet steam flow and (in contrast to many procedures reported in the literature) full details of the polydispersed droplet size spectrum following nucleation are retained in the calculations. The last aspect is essential for accurate modelling of the nucleation zone. This has been possible, without consuming excessive CPU time, by developing a novel averaging procedure that constantly redefines the average size and droplet number in each droplet group. In this way, the number of droplet groups required is restricted to an affordable optimum, while always retaining the correct shape of the droplet size spectrum.

A mixed Eulerian-Lagrangian technique is used. The continuity and momentum equations are solved by Denton's time-marching method. (The wet-steam "black-box" being flexible and modular, any other Eulerian time-marching method, e.g. Jameson's scheme, can be used.) The "black-box" performs the integration of the droplet growth equations along the fluid path lines rather than the usual, quasi-unsteady, method in which the pressure field remains frozen at a given instant of time while the growth of the liquid phase is calculated. The present scheme allows simultaneous solution of all the relevant equations and enforces the correct coupling between the vapour-phase gasdynamics and the relaxation effects due to the presence of the liquid phase.

For a proper comparison between experiments and theory, variation in pressure as well as droplet size must be considered. Many references compare the variation in pressure only. Such comparison is an inadequate test for nucleation and droplet growth theories. Almost any nucleation theory can be 'tuned' to reproduce the measured pressure distribution. A crucial test is to find out whether the same 'tuning' can predict the correct droplet size as well. Experience with calculations for wet steam points out categorically that, in general, predicting a satisfactory pressure distribution does not automatically ensure a good prediction of droplet size. The present computational scheme has been validated against measurements of steady (both sub- and supercritical) and unsteady condensation shock waves (Guha \& Young 1991). Various regimes of condensation have been computed by Guha (1994a), which shows a novel example of subsonic condensation where the nozzle is unchoked at the geometric throat. (In most reported studies on condensation shocks in nozzles, condensation takes place in the supersonic divergent part.)

Figure 1 presents one example of unsteady calculation. The prediction compares well with measurement. The pressure profiles at different instants during a complete cycle reveal exactly the same sequence of the formation and movement of the shock wave as explained earlier. As the aerodynamic shock wave moves upstream towards the throat and interacts with the nucleation zone, progressively fewer droplets are nucleated, thus resulting in a 


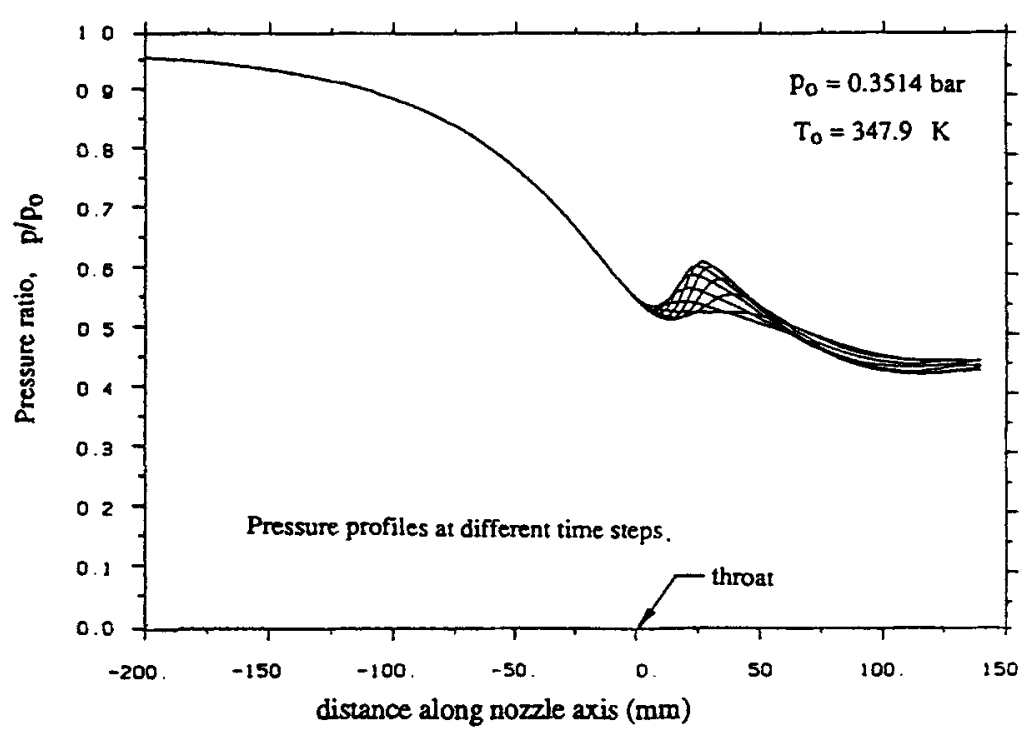

Figure 1. Unsteady condensation: Evolution of pressure for one cycle.

larger final mean radius. This causes a large variation in the droplet sizes during each cycle. An interesting implication of the unsteady nucleation process is that it may be a contributing factor to the formation of the highly-skewed polydispersed droplet spectrum measured in a real steam turbine: a polydispersity which cannot be predicted with steady flow calculation methods (see $\S 4$ ). Details of this and other calculations are in Guha \& Young (1991) and Guha (1994a).

\subsection{Integral analysis: Condensation wave theory}

A great deal of physics may be learnt from an integral analysis, which is a study of the jump conditions relating the end states of the condensation zone, without considering the detailed flow structure within it. Figure 2 shows an example calculation of the condensation wave theory for air with $1 \%$ moisture content. Guha (1994a) presents condensation wave

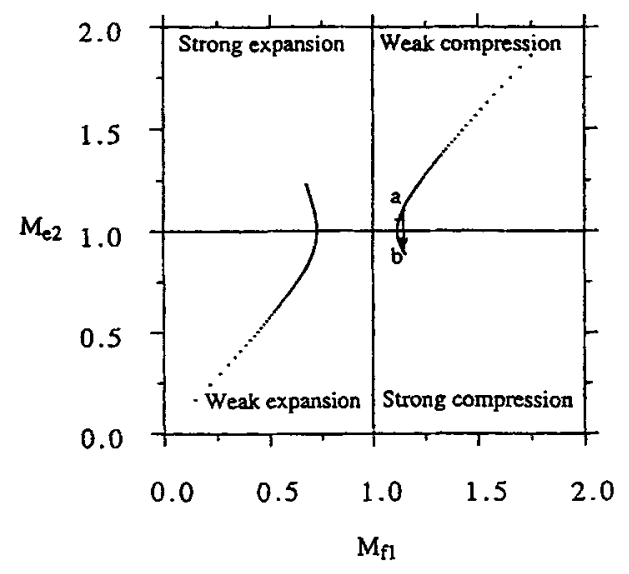

Figure 2. Condensation wave theory. 
theory in great detail and discusses similarities and differences with the more familiar combustion wave theory. (The reference considers control volume analysis of two types of near-discontinuities, condensation shock as well as aerodynamic shock waves in vapourdroplet flow, from a common standpoint.) In figure 2, $\mathbf{M}_{f 1}$ represents the upstream Mach number based on the frozen speed of sound $a_{f}$ and $\mathrm{M}_{e 2}$ represents the downstream Mach number based on the equilibrium speed of sound $a_{e}(\$ 3.2)$. The effect of heat release is very pronounced when the Mach number lies approximately between 0.8 and 1.1, and a small amount of condensation may alter the flow velocity etc., quite dramatically. Details may be found in Guha (1994a).

\subsection{Thermal choking due to non-equilibrium condensation}

It is well known that heat addition causes a reduction in Mach number in supersonic flow and an increase in Mach number in subsonic flow. In other words, heat addition to a flowing fluid drives the Mach number towards unity. Therefore, at a particular flow Mach number, the fluid can absorb a maximum quantity of heat before the local Mach number equals unity and the flow becomes thermally choked. Any elementary textbook on classical gas dynamics gives the expression for critical quantity of heat, $q_{\text {classical }}^{*}$, for simple heat addition to an ideal gas (external heat addition without any change in flow cross-sectional area).

Similar to the case of external heat addition, the Mach number decreases in the condensation zone (the flow being supersonic). Therefore, for particular combinations of nozzle geometry, supply conditions and the working fluid, the liberation of latent heat could be such that the minimum Mach number becomes unity and the flow is thermally choked. (A numerical computation of this limiting case of thermal choking due to non-equilibrium condensation is later shown in figure 4.) If the inlet total temperature, $T_{o 1}$, is reduced any further, keeping the inlet total pressure, $p_{o 1}$, fixed, continuous variation of the flow variables is no longer possible and an aerodynamic shock wave appears inside the condensation zone.

Although widely referred, it has been argued in Guha (1994b) that the expression for $q_{\text {classical }}^{*}$ is not appropriate for a condensing flow primarily for two reasons: (i) In case of a condensation shock, the heat is added as a result of condensation of a part of the fluid itself. Therefore, the mass flow rate of the condensable vapour changes as the vapour is continually transformed into the liquid phase. The expression for $q_{\text {classical }}^{*}$, which is derived for external heat addition to an ideal gas, does not take into account this mass depletion. (ii) The droplets formed through homogeneous nucleation grow at a finite rate by exchanging mass and energy with the surrounding vapour. Therefore, the energy addition due to condensation is not instantaneous and takes place over a short but finite zone. Since condensation shock normally occurs in the diverging section (with dry vapour at inlet), this means the flow area increases between the upstream $\left(A_{1}\right)$ and downstream $\left(A_{2}\right)$ of the condensation zone. The expression for $q_{\text {classical }}^{*}$, on the other hand, is derived by assuming heat addition in a constant area duct.

Guha (1994a) gives details about the relative importance of the above two effects. Two new expressions for critical quantity of heat have been derived: $q_{A}^{*}$ which deals with external heat addition with area variation, and $q_{\text {integral }}^{*}$ which takes into account the area variation as well as the depletion in the mass of vapour due to condensation. The dotted 


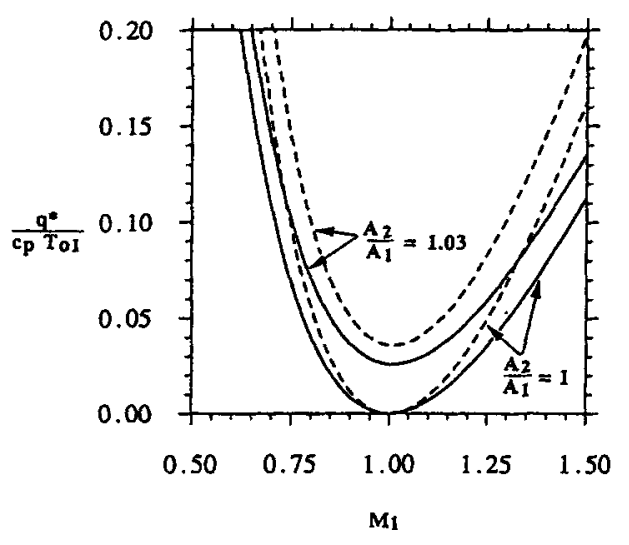

Figure 3. Effects of area ratio across the condensation zone and the depletion in vapour mass flow rate on the critical amount of condensation $\left(-q_{A}^{*}, \ldots\right.$ $q_{\text {integral }}^{*}$.

lines in figure 3 represent $q_{\mathrm{integral}}^{*}$ while the solid lines are the plots of $q_{A}^{*}$. Note that the solid line corresponding to unity area ratio $\left(A_{2} / A_{1}=1\right)$ is nothing but the classical solution, $q_{\text {classical }}^{*}$. Figure 3 shows clearly that the effects of mass depletion and (even a small) area variation are quite dramatic, especially when the Mach number is close to unity.

Figure 4 presents a time-marching solution of a limiting condensation shock, giving the variation in Mach number $(\mathrm{M})$, non-dimensional pressure $\left(p / p_{o 1}\right)$ and non-dimensional wetness fraction $\left(y / y_{e q}\right)$ along the nozzle axis. There are two sonic points in the flow field. (Achieving exactly a Mach number of unity at the end of the condensation zone (point 2) required numerical experiments of varying $T_{o 1}$ keeping $p_{o 1}$ fixed.) At the conditions shown in figure $4 A_{2} / A_{1}=1.0284, \mathrm{M}_{1}=1.182$. The critical amount of heat obtained from this full numerical solution of the nozzle flow is denoted by $q_{\text {actual }}^{*}$. Table 1 compares the results of various integral analyses.

In conclusion, a theory of thermal choking due to non-equilibrium condensation in a nozzle is presented (Guha 1994b). The theory is based on a simple control volume approach. (A differential theory of thermal choking is discussed in Guha (1994a).) It applies to vapour-droplet flow with or without a carrier gas. The expression for critical heat (or condensation) derived is valid for either supersonic or subsonic flow, and for heat release either in the diverging or in the converging part of a nozzle.

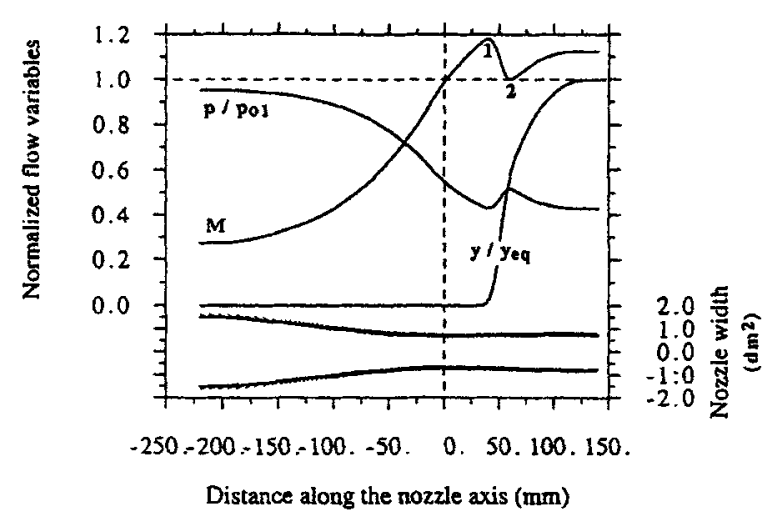

Figure 4. Time-marching solution of a limiting condensation shock in a quasi-onedimensional convergent-divergent nozzle. (Continuous variation of flow variables leading to thermal choking at point 2.) 
Table 1. Comparison of integral predictions with exact solution.

\begin{tabular}{|c|c|c|c|}
\hline \multicolumn{2}{|c|}{ External heat addition to ideal gas } & \multirow{2}{*}{$\begin{array}{l}\text { Condensational heat } \\
\text { release with area } \\
\text { variation and mass } \\
\text { depletion of vapour } \\
\left(q_{\text {integral }}^{*} / c_{p g} T_{o 1}\right)\end{array}$} & \multirow{2}{*}{$\begin{array}{l}\text { Time-marching solution } \\
\text { of non-equilibrium } \\
\text { gas dynamic equations } \\
\left(q_{\text {actual }}^{*} / c_{p g} T_{o 1}\right) \\
\end{array}$} \\
\hline $\begin{array}{l}\text { in constant area duct } \\
\left(q_{\text {classical }}^{*} / c_{p g} T_{o 1}\right)\end{array}$ & $\begin{array}{c}\text { in duct of varying area } \\
\left(q_{A}^{*} / c_{p g} T_{o 1}\right)\end{array}$ & & \\
\hline 0.01978 & 0.0423 & 0.0574 & 0.0587 \\
\hline
\end{tabular}

Table 1 shows that the present theory, $q_{\text {integral }}^{*}$, is in very close agreement with the full numerical solution of the differential equations of motion (giving the detailed structure of a condensation shock wave leading to thermal choking). The usually quoted $q_{\text {classical }}^{*}$ underestimates the critical heat by a factor of three in the example calculation presented. The variation of area across the condensation zone (although small) and the depletion in vapour mass as a result of condensation cannot be neglected in determining the critical heat in condensing nozzle flow.

\section{Fluid dynamics with interphase transport of mass, momentum and energy in pure vapour-droplet mixtures}

\subsection{Relaxation gas dynamics for vapour-droplet mixtures}

A lucid description covering many aspects of relaxation gas dynamics and its applications to vapour-droplet flows (including coupled relaxation processes) may be found in Guha (1995).

If a property of a medium is perturbed from its equilibrium state and the restoration to equilibrium occurs at a finite rate, the medium is called a relaxing medium and the process of restoration is termed relaxation. Simple relaxing media follow the archetypal rule of the restoration process following a perturbation: $d(\Delta q) / \mathrm{d} t=-\Delta q / \tau$, where, $q$ is an internal state variable, $\Delta q$ is the departure from equilibrium and $\tau$ is the relaxation time.

A vapour-droplet medium is assumed to be a homogeneous two-phase mixture of a large number of fine, spherical droplets dispersed in the continuous vapour phase. Although the droplet cloud may exhibit an arbitrary level of polydispersity with a spectrum of different sizes of droplets, in the present analysis we will restrict ourselves to a mono-dispersed droplet population for simplicity of description. We also consider pure substances only. The droplets are large so that the capillary subcooling is negligible.

A vapour-droplet medium may go out of equilibrium in three different ways as below.

(i) The droplet temperature is not equal to the saturation temperature $\left(T_{l} \neq T_{s}\right)$. $\Delta T_{l}=T_{s}-T_{l}$ is the relevant non-equilibrium variable and $\tau_{D}$ is the corresponding droplet temperature relaxation time.

(ii) The two phases have unequal velocities, i.e., there may be a slip between the phases $\left(V_{g} \neq V_{l}\right) . \Delta V=V_{g}-V_{l}$ is the non-equilibrium variable and $\tau_{I}$ is the corresponding inertial relaxation time. 


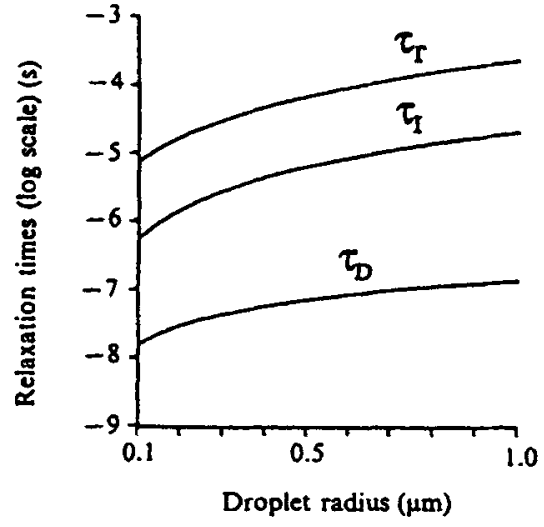

Figure 5. Relaxation times for water droplets in pure steam.

(iii) The temperature of the vapour phase may be different from the saturation temperature $\left(T_{g} \neq T_{s}\right) . \Delta T=T_{s}-T_{g}$ is the relevant non-equilibrium variable and $\tau_{T}$ is the corresponding vapour thermal relaxation time. [Note that negative $\Delta T$ means that the vapour is superheated.]

It is clear that under non-equilibrium conditions mass, momentum and energy transfers would take place between the two phases. It is through these interphase transfer processes that the system returns to equilibrium following a perturbation. The equations representing the different interphase transfer processes and the expressions for $\tau_{D}, \tau_{I}$ and $\tau_{T}$ have been derived in Young \& Guha (1991) and Guha (1995). Figure 5 shows the variation of the three relaxation times in pure steam $(p=0.5 \mathrm{bar}, y=0.1)$ as a function of droplet radius.

It can be shown (Guha 1992c) that, quite generally, the relation $\tau_{D} \ll \tau_{I} \ll \tau_{T}$ holds in pure vapour-droplet flow. Therefore, following a disturbance, on a very short time scale the droplet temperature reaches equilibrium, then the velocity slip and finally the vapour temperature relaxes to the equilibrium value. While considering any particular relaxation process, therefore, relaxation processes with smaller relaxation times may be assumed to have been equilibrated whereas relaxation processes with larger time scales may be assumed to be frozen. Mathematically, for example, equilibrium droplet temperature means that $\Delta T_{l}$ and $\tau_{D}$ simultaneously tend to zero, the ratio $\Delta T_{l} / \tau_{D}$ remaining finite such that $\Delta T_{l} / \tau_{D} \rightarrow V_{l}\left(\mathrm{~d} T_{s} / \mathrm{d} x\right)$, and, for instance, frozen heat transfer implies $\tau_{T} \rightarrow \infty$.

\subsection{Speeds of sound in a pure vapour-droplet mixture}

As a result of three relaxation processes in vapour-droplet flow, four different sound speeds $\left(a_{f}, a_{e 1}, a_{e 2}, a_{e}\right)$ may be defined subject to different mechanical and thermodynamic constraints. The full frozen speed of sound, $a_{f}$, corresponds to the speed of an harmonic acoustic wave of such high frequency that the response of the droplets is negligible (i.e. zero mass, momentum and energy transfer). The full equilibrium speed of sound, $a_{e}$, corresponds to the speed of an harmonic acoustic wave of such low frequency that liquidvapour equilibrium is maintained at all times. The two intermediate speeds correspond to (a) the case of equilibrium droplet temperature relaxation but frozen momentum and 


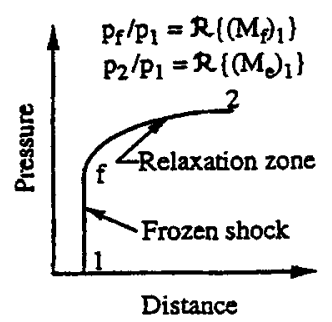

Partly dispersed wave

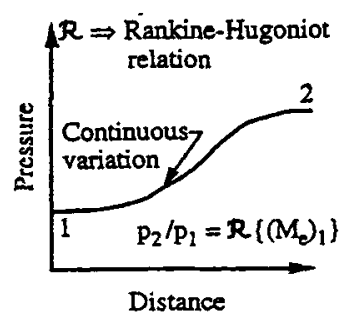

Fully dispersed wave
Figure 6. Schematic structure of shock waves. (a) Partly and (b) fully dispersed waves.

heat transfer $\left(a_{e 1}\right)$, and (b) the case of equilibrium droplet temperature and velocity slip relaxation but frozen heat transfer $\left(a_{e 2}\right)$.

The derivation and the expressions of the sound speeds may be found in Young \& Guha (1991) and Guha (1995). The relationship of the four sound speeds to each other is of great importance. As a typical example, the ratios of different sound speeds for steam at $1 \mathrm{bar}$ and 0.1 wetness fraction are given by, $a_{f}: a_{e 1}: a_{e 2}: a_{e} \equiv 1: 0.997: 0.945: 0.878$.

\subsection{Structure of stationary shock waves}

One can define at least two Mach numbers corresponding to the limiting speeds of sound: a frozen Mach number $M_{f}=V / a_{f}$, and an equilibrium Mach number $\mathrm{M}_{e}=V / a_{e}$, where $V$ is the flow velocity. In general, $a_{e}<a_{f}$ and $\mathbf{M}_{e}>\mathbf{M}_{f}$. As a result of the frequency dispersion, therefore, two distinct types of shock waves might form in a relaxing medium, the far upstream and far downstream conditions being at equilibrium (figure 6): (1) A partly dispersed shock wave, where a discontinuous jump in flow variables (dominated by viscosity and thermal conductivity as in Rankine-Hugoniot shocks in classical gas dynamics) is followed by a continuous relaxation zone in which the fluid returns to equilibrium by performing the relaxation processes. Such a case arises if the upstream velocity is greater than the frozen speed of sound, i.e., if $V_{g o}>a_{f o}$ or, $\mathbf{M}_{f o}>1$. In order to denote the upstream condition, we use both subscripts $o$ (so that confusion does not arise with sound speeds $a_{e 1}$ and $a_{e 2}$ ) and 1 (as this is the conventional nomenclature for Rankine-Hugoniot conditions discussed in \$3.5). (2) A fully dispersed shock wave, where a continuous change of flow properties takes place from the upstream to the downstream equilibrium state. This corresponds to the case when $a_{f o} \leq V_{g o}<a_{e o}$; i.e., when $\mathbf{M}_{f o} \leq 1$, but $\mathbf{M}_{e o}>1$.

With four limiting speeds of sound in a vapour-droplet mixture, four types of stationary shock wave structures may arise (Young \& Guha 1991; Guha 1992c, 1995):

$\begin{array}{llr}\text { type I waves } & \text { corresponding to } & a_{e o}<V_{g o}<a_{e 2 o}, \\ \text { type II waves } & \text { corresponding to } & a_{e 2 o}<V_{g o}<a_{e 1 o}, \\ \text { type III waves } & \text { corresponding to } & a_{e 1 o}<V_{g o}<a_{f o}, \\ \text { partly dispersed waves } & \text { corresponding to } & V_{g o}>a_{f o} .\end{array}$

Type I, II and III waves are sub-categories of the fully dispersed waves, where the steepening effect of the nonlinear terms in the equations of motion is just balanced by the dispersive effect of the relaxation processes. Type I waves are dominated by vapour thermal relaxation, type II waves by both velocity and vapour thermal relaxation and type III waves by all three relaxation processes. It has been shown (Young \& Guha 1991; Guha 1995) 
that $\left|\Delta T_{l}\right|$ becomes unstable in the interval $a_{e 1}<V_{g}<a_{f},|\Delta V|$ becomes unstable in the interval $a_{e 2}<V_{g}<a_{e 1}$, and, $|\Delta T|$ becomes unstable in the interval $a_{e}<V_{g}<a_{e 2}$. These instabilities in the non-equilibrium variables are the reasons for the existence of the fully dispersed waves. Figure 7 shows numerical solutions for a typical type II fully dispersed wave in a steady flow of pure wet steam. Numerical solutions of other types of waves may be found in (Guha \& Young 1989; Guha 1992b, 1995).

The usual model of a partly dispersed shock wave assumes that the interphase transfer processes are frozen during the passage through the discontinuity and the vapour properties just downstream of the discontinuity can be calculated using a standard Rankine-Hugoniot analysis. The liquid droplets therefore pass through the frozen shock without change in radius, temperature and velocity. The conditions downstream of the discontinuity provide the initial values for integrating the two-phase conservation equations through the relaxation zones. The droplet temperature relaxes very quickly, followed by velocity slip and finally the vapour temperature. The lengths of the relaxation zones are in the approximate ratios $\tau_{D}: \tau_{I}: \tau_{T}$. Details about partly dispersed waves in vapour-droplet flow may be found in (Guha 1992b). Although linearized analyses are often presented for the relaxation zone downstream of the frozen shock in standard treatments on relaxation gas dynamics, they are of limited applicability to vapour-droplet flows (Guha 1992b).

\subsection{Shock waves in unsteady flow}

The physical significance of the various wave profiles discussed above can be appreciated more readily by considering their development under unsteady flow conditions. As a typical example, we now discuss wave generation in one-dimensional flow by an instantaneously accelerated piston in a frictionless pipe initially containing stationary wet steam. The numerical scheme and other details may be found in Guha \& Young (1989).

Figure 8 shows the numerical prediction of a wave propagating in wet steam. The figure also includes the flow behaviour in a dry, ideal gas under identical conditions in order to illustrate the special features of a vapour-droplet mixture. The $(t-x)$ diagram was constructed from the results of the unsteady time-marching calculation. At the instant of initiation, all the interphase transfer processes are frozen and the shock velocity corresponds to the propagation velocity into a single-phase vapour at the same temperature. Behind the shock, the mixture relaxes to equilibrium along the particle pathlines. The droplet temperature relaxes first on the very short timescale $\tau_{D}$ and is followed by the velocity slip and vapour temperature on timescales $\tau_{I}$ and $\tau_{T}$ respectively. Changes along the particle paths are propagated upstream and downstream along the left and right running Mach lines (based on the frozen speed of sound). The right running Mach lines overtake the shock wave, weakening it and causing it to slow down. The shock path therefore curves in the $(t-x)$ diagram until it reaches a constant equilibrium speed. When this occurs, the dispersive effects of the relaxation processes are just balanced by the steepening effects of the nonlinear terms and the wave structure is identical to that of the stationary waves in steady flow described earlier. Whether the final equilibrium structure is partly or fully dispersed depends on the piston velocity (for analytical conditions, see Guha 1992c, 1995).

The variation of the wave pressure profile with time is also shown in figure 8 and the deceleration and weakening of the wave front are clearly visible. The behaviour of the 

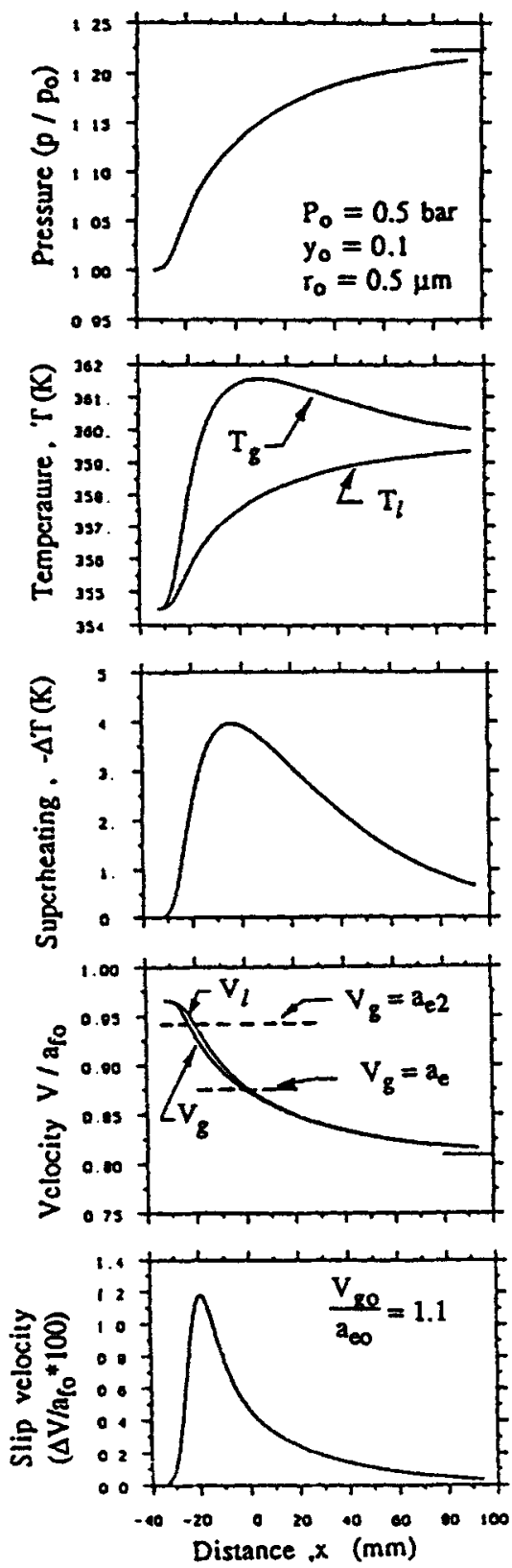

Figure 7. Numerical solution for a Type II wave in wet steam. 

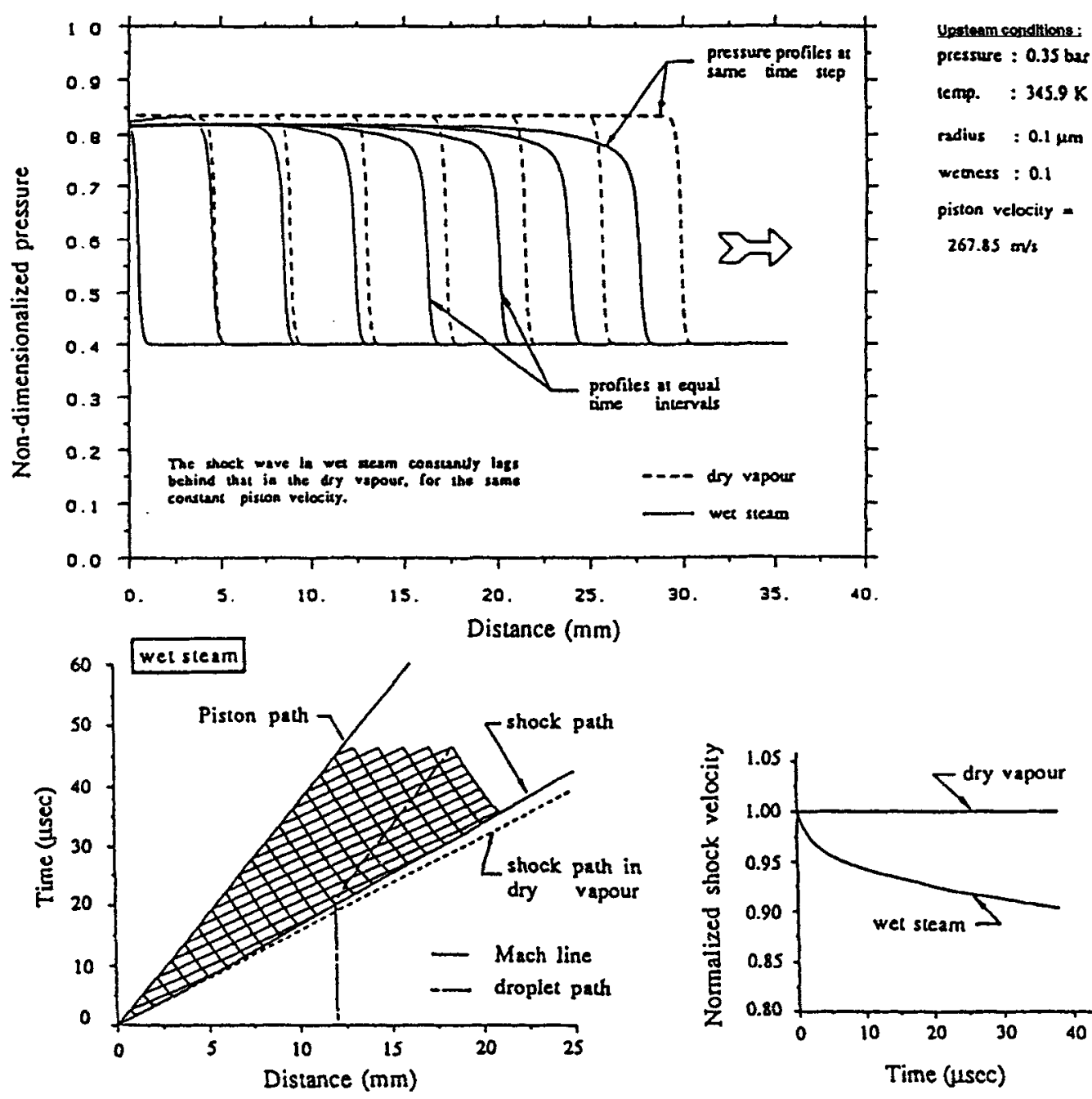
pressure : 0.35 bas

- prome profiles as .

radius : $0.1 \mathrm{\mu m}$

we oness : 0.1
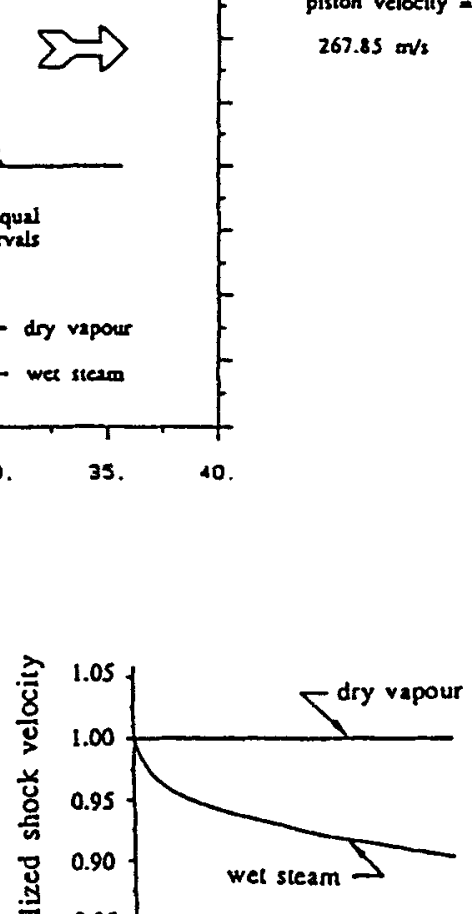

Figure 8. Numerical prediction of shock propagation in a pipe.

superheat vapour temperature, droplet radius and wetness fraction is shown by the curves in figure 9 which are self-explanatory. As with stationary partly dispersed shock waves, the increase in wetness fraction downstream of the frozen shock wave is due to the effects of velocity slip.

\subsection{Integral analysis: Jump conditions}

Detailed study on the jump conditions across shock waves has been made by Guha (1992a, 1994a) and the similarities and differences of condensation discontinuities and aerodynamic shock waves are discussed at length (Guha 1994a). Here, we can only mention the bare minimum.

In a simple relaxing medium, e.g., a solid-particle-laden gas, the form of the jump conditions ( $\mathrm{R}$ in figure 6 ) are identical to the Rankine-Hugoniot relations for an ideal gas, 

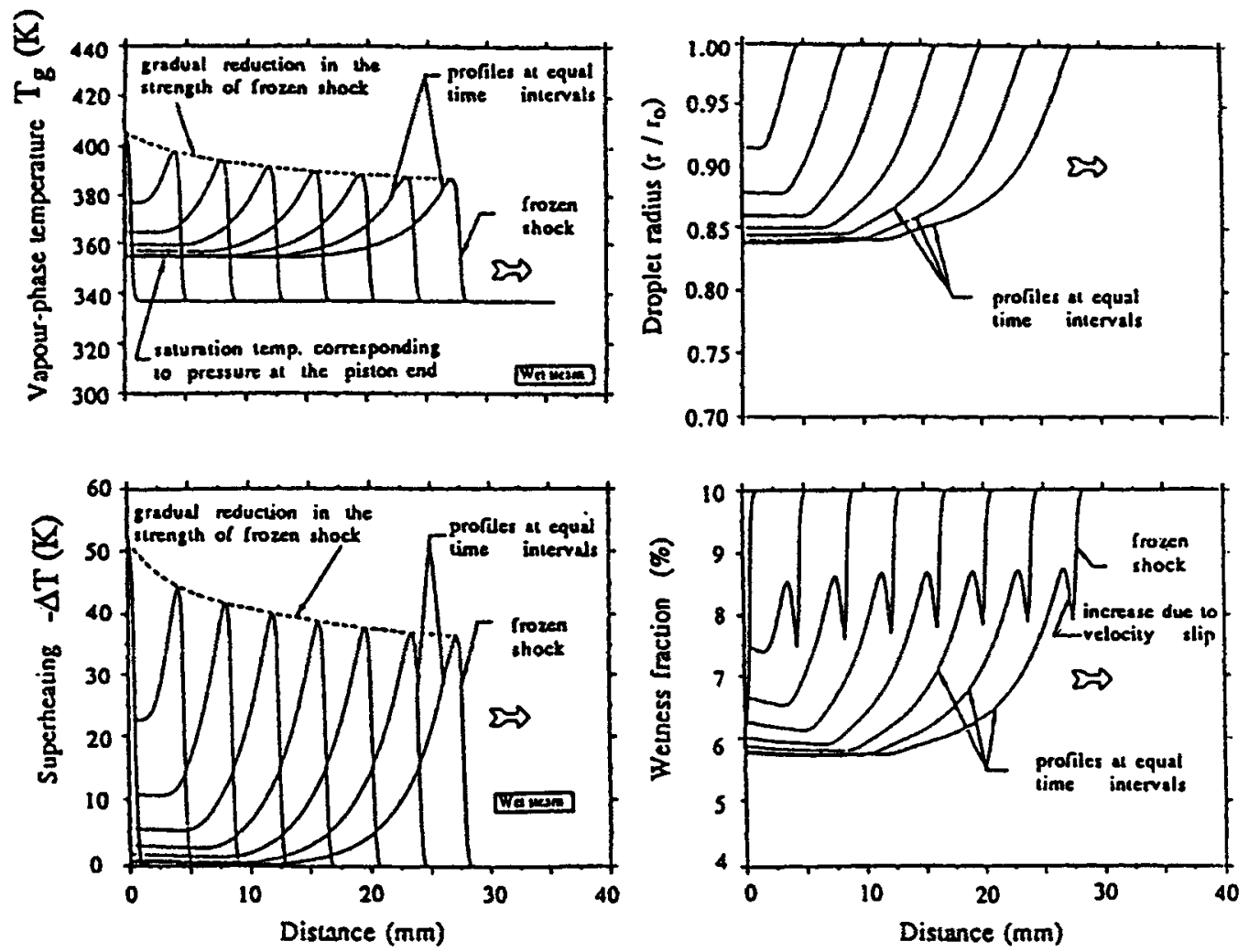

Figure 9. Variation of flow parameters during shock propagation in wet steam.

if one uses the equilibrium Mach number, $\mathbf{M}_{e}$, and the equilibrium isentropic index, $\gamma_{e}$. (Expressions for $\gamma_{e}$ in vapour-droplet mixtures with or without a carrier gas are given in Guha 1992a, 1994a) However, it has been shown by Guha (1992a) that although jump conditions of the same form as Rankine-Hugoniot relations can be formulated for vapourdroplet mixtures, they are approximate and hold only conditionally.

A solid-particle-laden gas can be treated as a modified gas. Equations governing vapourdroplet flow are much more complex. An additional complexity (and novelty) occurs due to interphase mass transfer (the liquid phase evaporates inside a dispersed wave). If the strength of the wave is sufficient, complete evaporation may result, thereby rendering a two-phase medium before a shock wave into a single-phase one after it!

The integral analysis (Guha 1992a) reveals that, depending on the upstream wetness fraction and the pressure ratio across the wave, four types of shock structures may result in vapour-droplet flow. They are: (I) equilibrium fully dispersed, (II) equilibrium partly dispersed, (III) fully dispersed with complete evaporation, (IV) partly dispersed with complete evaporation. Figure 10 shows the boundaries of the four regimes in low-pressure wet steam.

Jump conditions across all types of aerodynamic waves are derived by Guha (1992a, 1994a). Figure 11 shows the predictions of the integral analysis compared with numerical solutions of the wave profile as discussed in $\$ 3.3$. The dotted lines in figure 11 represent the jump conditions. The solid lines represent numerical solutions for three 


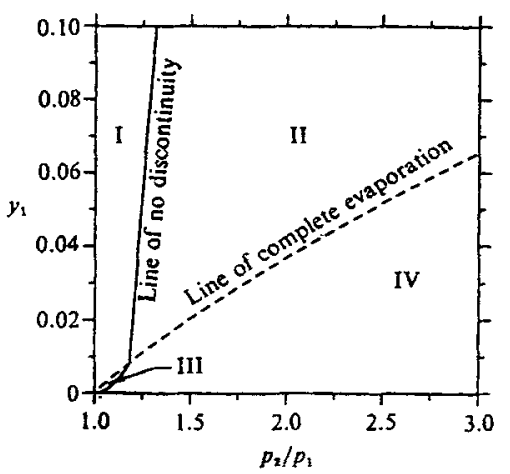

Figure 10. Phase diagram of different shock structures in low-pressure steam.

categories of shock waves: (I), partly dispersed with complete evaporation ( $p_{1}=0.35$ bar, $\left.\mathrm{M}_{f 1}=1.6, y_{1}=0.05, r_{1}=0.1 \mu \mathrm{m}\right)$; (II) equilibrium partly dispersed ( $p_{1}=0.35 \mathrm{bar}$, $\left.\mathbf{M}_{f 1}=1.2, y_{1}=0.05, r_{1}=0.1 \mu m\right)$; (III) equilibrium fully dispersed ( $p_{1}=0.35$ bar, $\mathrm{M}_{f 1}=0.97, y_{1}=0.1, r_{1}=0.1 \mu m$ ). Results from integral analyses agree remarkably well with solutions of the differential equations of motion, thereby confirming independent theoretical consistency.

It is interesting to study the various mechanisms of entropy creation inside a dispersed shock wave (Guha 1992a). In addition to the effects of viscosity and thermal conduction, entropy is created due to the relaxation processes. Figure 12 shows the rise in entropy across shock waves as a function of upstream frozen Mach number. Two cases are considered: dry steam, and, wet steam with upstream wetness fraction 0.1 . Shock waves may occur in wet steam even when $\mathbf{M}_{f 1}<1$, due to the existence of fully dispersed waves. In a partly dispersed wave, entropy rises across the frozen shock as well within the relaxation zone that follows. The figure shows that the contribution of the relaxation processes is very significant in the overall creation of entropy.

In an ideal gas, the total rise in entropy across a shock wave is fixed by RankineHugoniot relations, the magnitudes of viscosity and thermal conductivity merely determine the thickness of the shock wave. It has been shown (Guha 1992a) that the total rise in entropy across a dispersed shock wave in vapour-droplet mixtures is similarly fixed by the jump conditions. In exact analogy with the role of viscosity, various relaxation processes and

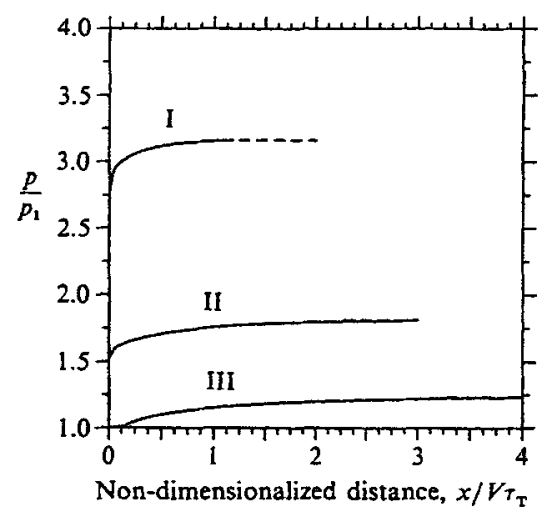

Figure 11. Comparison of asymptotic pressure profiles with generalised Rankine-Hugoniot equations. 


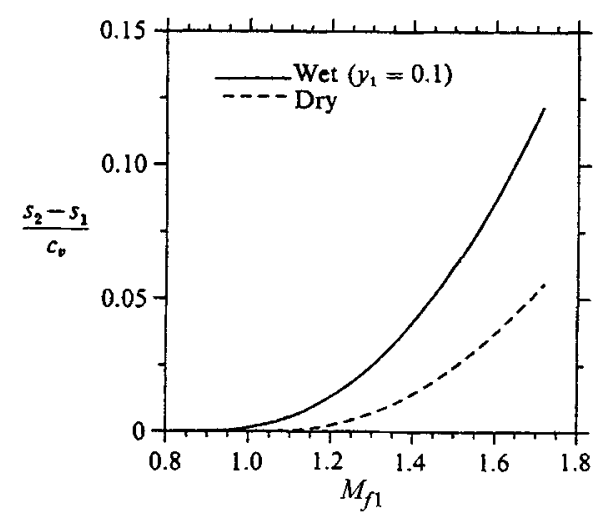

Figure 12. Entropy rise across shock waves in low-pressure steam.

their timescales determine the thickness of the shock wave. Overall jump conditions in any property, including entropy, can be determined without any explicit reference to the processes which make the 'jump' happen!

An integral analysis as well provide much insight into the unsteady development of shock waves. Details can be found in (Guha 1995; Guha 1992c).

\subsection{Interpretation of total pressure and temperature in two-phase flow}

In this section we discuss briefly some interesting effects of the non-equilibrium, interphase transfer mechanisms in a stagnation process in two-phase flow. Pitot measurements are often used for inferring velocity or loss (entropy generation) in multiphase mixtures. In single-phase fluids, the fluid is assumed to be brought to rest at the mouth of the Pitot tube isentropically. Hence flow Mach number and entropy generation (in steady, adiabatic flow) are uniquely determined by the total pressure measured by a Pitot tube, together with an independent measurement of the static pressure. (In supersonic flow in an ideal gas, application of Rankine-Hugoniot equations across the detached shock wave in front of a Pitot tube retains the utility of Pitot measurements for deducing flow Mach number and entropy generation.) Pitot measurements in a multiphase mixture, however, require careful considerations. Similar considerations are also needed for interpreting total temperature. Guha (1997a) gives the details of the physical considerations required and the description of a unified theory for the interpretation of total pressure as well as total temperature.

The solid particles or the liquid droplets respond to changes in temperature, velocity etc. of the gas phase through interphase exchanges of mass, momentum and energy. These are essentially rate processes and hence significant departures from equilibrium can take place if the rate of change of external conditions, imposed by the deceleration in the stagnating flow, is comparable to the internal time scales. Thus, for example, if the size of the liquid droplets or the solid particles is very small, then inertial and thermodynamic equilibrium between the two phases are maintained always, and a Pitot tube would measure the equilibrium total pressure, $p_{o e}$. On the other hand, if the size of the droplets or the particles is very large, all interphase transfer processes remain essentially frozen. The Pitot tube records the pressure which it would have recorded if the vapour phase alone was brought to rest from the same velocity. The total pressure in this case is termed the 
frozen total pressure, $p_{o f}$. Analytical expressions for $p_{o e}$ and $p_{o f}$, both in vapour-droplet and gas-particle flow, are given (Guha 1997a).

As an example, consider low-pressure wet steam with a typical wetness fraction of $10 \%$ and at a Mach number 1.5. Calculations show that $p_{o f} / p=3.3$ and $p_{o e} / p=3.79$, where $p$ is the static pressure. Therefore, in this particular example, the equilibrium total pressure is about $15 \%$ higher than the frozen total pressure.

It is expected that for intermediate sizes of the droplets or particles, the pressure recorded by the probe would neither be the equilibrium nor the frozen value. The imposed deceleration in front of the Pitot tube would cause the two-phase mixture to deviate from equilibrium conditions, both inertially and thermodynamically. The deceleration process consequently ceases to be isentropic, as non-equilibrium exchanges of mass, momentum and energy between the two phases create entropy.

Guha (1997a) has considered a large number of two-phase mixtures, both gas-particle and vapour-droplet, at subsonic as well as supersonic velocities for many different sizes of the droplets (or particles). In the supersonic case a detached frozen shock wave stands in front of the Pitot tube. The relaxation mechanisms in a gas-particle mixture are different from those in a vapour-droplet flow. Despite all these complexities and differences, it was possible with proper non-dimensionalization of flow parameters to adopt a universal plot, within acceptable tolerance, of non-dimensional total pressure, $R_{p}$, versus Stokes number, St (which is a non-dimensional representation of particle size). $R_{p}$ and St are defined by, $R_{p} \equiv\left(p_{o}-p_{o f}\right) /\left(p_{o e}-p_{o f}\right)$ and $\mathrm{St} \equiv \tau_{I} V_{\infty} / L$, where, $V_{\infty}$ is the unperturbed velocity of the two-phase mixture far upstream of the measuring device, $p_{o}$ is the pressure attained at the measuring point under non-equilibrium conditions (the total pressure which is measured) and $L$ is a characteristic length (in subsonic flow $L$ is related to the Pitot diameter, in supersonic flow $L$ is related to the distance between the frozen shock wave and the Pitot mouth). Larger droplets or particles correspond to higher St.

Figure 13 shows the variation of $R_{p}$ with $\mathrm{St}$, which may be adopted as the Pitot correction curve usable at a wide range of subsonic and supersonic Mach numbers and for any two-phase mixtures (vapour-droplet or gas-particle). The variation is monotonic. It should be noted that the denominator in the expression for $R_{p}$ is calculated using the equilibrium thermodynamics, whereas the numerator is calculated using non-equilibrium equations.

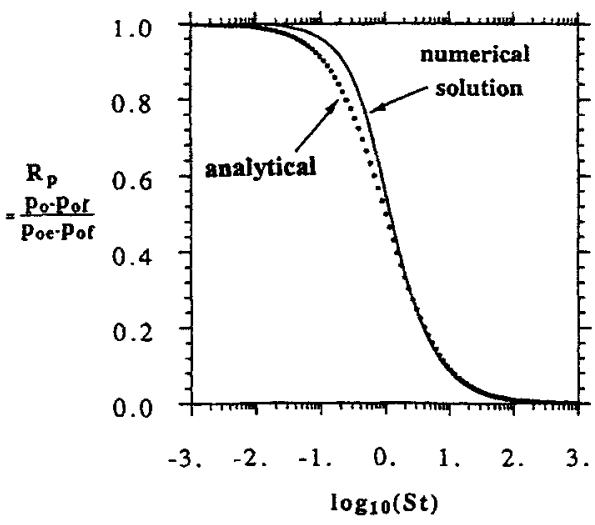

Figure 13. Near-universal plot of $R_{p}$ versus St: comparison of a simple analytical theory with numerical solutions. 


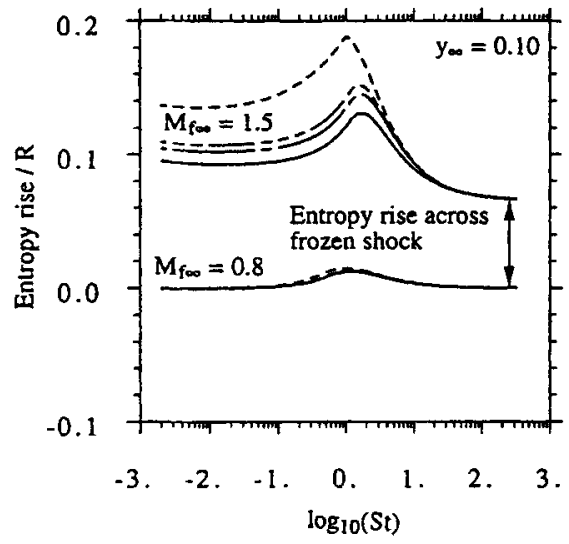

Figure 14. Entropy rise versus Stokes number in air with different solid particles $\delta=0.1(-), 0.8(---), 1.2(---)$, $4.0(-)$.

That the value of $R_{p}$, shown in figure 13 , indeed tends to unity and zero in the appropriate limits of $\mathrm{St}$, demonstrates independent theoretical consistency of the calculation schemes.

In addition to these numerical calculations, an analytical theory for determining total pressure under non-equilibrium conditions has been formulated. The analytical theory is simple and is amenable to direct physical interpretation. The theory shows that $R_{p}=1 /(1+\mathrm{St})$. The predicted total pressure correctly reduces to the frozen total pressure in the limit of large Stokes number (large particles) and to the equilibrium total pressure in the limit of small Stokes number (small particles). Maximum dependence of the total pressure on Stokes number is observed when the Stokes number is of the order unity. The analytical result is also plotted in figure 13 for comparison. Under non-equilibrium conditions for intermediate $\mathrm{St}$, the prediction of this equation compares very well with results from full numerical solution of the gas dynamic equations for two-phase mixtures.

Figure 14 plots the rise in mixture entropy, as mixtures of air and solid particles are decelerated by a measuring probe from their far upstream velocity to rest. Four different solid particles (hypothetical) with $\delta \equiv c_{l} / c_{p g}=0.1,0.8,1.2$ and 4 are considered and the calculations are done for two Mach numbers. For the subsonic case $\left(\mathbf{M}_{f \infty}=0.8\right)$, figure 14 shows that the rise in entropy is indeed maximum when $\mathrm{St} \sim 1$, and is almost zero in the frozen and equilibrium limits. (Recall from figure 13 that the total pressures are different in these limits.) At $\mathbf{M}_{f \infty}=1.5$, the entropy rise is again maximum close to $\mathrm{St} \sim 1$, but it has a finite value both at $\mathrm{St} \rightarrow 0$ and at $\mathrm{St} \rightarrow \infty$. The rise in entropy in the limit $\mathrm{St} \rightarrow \infty$ is simply that across the frozen shock. (Since the same frozen shock is involved in all cases because the same $\mathbf{M}_{f \infty}$ is used, this increase in entropy is the same for all four mixtures considered.) The rise in entropy in the limit St $\rightarrow 0$ is, however, different for different mixtures (it depends on the isentropic index of the mixture and hence on $\delta$ ). However, it is shown (Guha 1992a) that if the particles come to equilibrium downstream of a frozen shock wave, then the entropy rise (across the shock plus the relaxation zone) is not dependent on the particle size (and hence on the relaxation times) but is determined completely by Rankine-Hugoniot equations for two-phase flow. This fact is reflected in the straight, horizontal portions of the curves (at $\mathrm{M}_{f \infty}=1.5$ ) in figure 14 in the limit St $\rightarrow 0$. 
The rate of entropy production in a multiphase mixture is maximum when the Stokes number is of the order unity (in accordance with other results of relaxation gas dynamics), and a reduction in measured total pressure is not unequivocally related to a rise in entropy (as it is in steady, adiabatic flow of single-phase fluids). The fact that the total pressure decreases monotonically from $p_{o e}$ to $p_{o f}$ as St changes form 0 to $\infty$ whereas the entropy rise is zero at both limits and has a maxima when $\mathrm{St} \sim 1$, demands care while interpreting Pitot measurements in multiphase flow.

\section{Application of the non-equilibrium theory to steam turbines}

\subsection{The formation of the liquid phase}

An introduction to the flow through steam turbines may be found in Guha (1995). In a multistage steam turbine used in power plants for generating electricity, the steam enters the low-pressure (LP) turbine cylinders as a dry superheated vapour but becomes wet towards the last stages. Experiments show that the water in turbines exists in two quite different forms. Usually more than $90 \%$ of the mass is concentrated in the fog, which consists of a very large number of very fine droplets (diameter $0.05-2.0 \mu \mathrm{m}$ ). The rest is in the form of coarse droplets which are very much larger (diameter 20-200 $\mu \mathrm{m}$ ). Coarse water is formed as a small proportion of the fog (typically $2-3 \%$ per blade row) is deposited on the blade surface either due to inertial impaction or through turbulent diffusion. The deposited water is drawn towards the trailing edge by the steam flow (or centrifuged towards the casing on moving blades), where it is re-entrained in the form of large droplets. The large droplets cause blade erosion, but their thermodynamic and mechanical effects on the steam flow can nearly always be neglected.

Formation of new droplets occurs only over a small part of the expansion in a turbine. As a fluid particle flows through the machine, typically the complete nucleation process takes only $10-20 \mu \mathrm{s}$, as compared with a typical flow transit time of 5-10 ms through an LP turbine. Thus most of the expansion in the turbine simply involves condensation on existing droplets. However, nucleation is of crucial importance as it is the process which establishes the final fog droplet size distribution which, in turn, determines the subsequent departure from equilibrium affecting the flow behaviour, the magnitude of the wetness loss and the rate of fog droplet deposition on the blading forming coarse water. Once the droplet size distribution can be accurately predicted, the analysis of the wet steam flow through the rest of the turbine rests on more solid foundation. However, currently no theory exists (see $\$ 4.2$ for a novel theory) which gives even remote agreement with the available experimental measurements of the size distribution of fog droplets in turbines.

Our inability to understand the nucleation process in steam turbines is surprising given the success with which spontaneous condensation in laboratory nozzles and stationary, two-dimensional, laboratory cascade of steam turbine blades can be predicted using a synthesis of the classical theories of homogeneous nucleation and droplet growth with the conservation equations of gas dynamics. Such calculations have now been refined to the extent that the theory, amended by only a modicum of empiricism, gives acceptable 


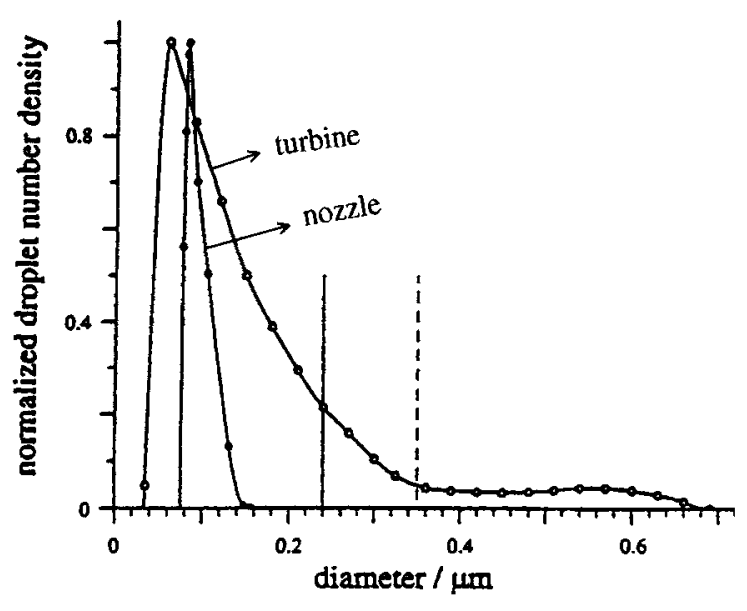

Figure 15. Typical droplet size distributions in a supersonic condensing steam nozzle (computed) and in the exhaust of a low pressure, electricity generating steam turbine (inferred from optical transmission data). - nozzle; ○ turbine ; - - - Sauter mean; - mass mean. (Turbine measurements courtesy of PT Walters, National Power Technology and Environment Centre, Leatherhead, UK.)

agreement in terms of pressure distribution and mean droplet diameter with most experiments reported in the literature.

As shown in figure 15, calculations of droplet size spectra in condensing steam nozzles usually indicate a narrow distribution with a comparatively small mean droplet diameter strongly dependent on the local expansion rate near the Wilson point. Because of the narrowness of the distribution, optical experimental techniques based on measuring the attenuation of light of different wavelengths are unable to resolve the details of the spectrum but register results consistent with a near monodispersed droplet population. In turbines, however, experimental determinations of droplet size spectra give quite different results, the optical characteristics of the medium invariably indicating a broad, strongly skewed, distribution with a much larger mean diameter, typically in the range $0.2-0.6 \mu \mathrm{m}$. Quite often, the distribution is bimodal with a significant proportion of the total mass of liquid contained in a secondary population of droplets having diameters in the range $0.4-1.0 \mu \mathrm{m}$. A typical distribution, inferred from light attenuation measurements in a low-pressure steam turbine used for electricity generation, is also shown in figure 15 .

Early attempts to describe the process of phase-transition in steam turbines followed the work of Gyarmathy who modelled the turbine by a series of one-dimensional nozzles. Reversion to equilibrium from the supersaturated vapour state was predicted to occur at a well-defined Wilson point in a particular blade passage giving rise to a near-monodispersed population of usually rather small droplets. The nucleation zone was thought to extend over a very short distance in the flow direction and calculations indicated that the mean droplet size should be very sensitive to turbine inlet conditions, small changes of which had the potential for displacing the Wilson point to new locations of quite different expansion rate. Later calculations of nucleation and droplet growth in two-dimensional turbine cascades displayed characteristics essentially similar to those found in one-dimensional condensation studies, albeit in more complicated and realistic fluid flow fields.

As noted above, optical measurements of wetness fraction and droplet spectra in turbines tell quite a different story. The measured spectrum is always broader, and the mean diameter larger, than conventional calculations indicate. Furthermore, the spectrum is comparatively insensitive to small changes in turbine inlet conditions: Measurements taken on the same machine over a period of years show excellent reproducibility. Finally, it appears 
that the reversion process does not occur in very narrow condensation zones as observed in laboratory nozzles but generally occupies much greater distances in the flow direction. This behaviour can be deduced from optical measurements which show that the wetness fractions at certain locations in some turbines are considerably lower than the local equilibrium values.

It is therefore evident that the nucleation of water droplets in turbines involves phenomena which are not reproduced by laboratory experiments on nozzles and stationary cascades but nevertheless play a dominating role in the process of phase-transition in real machines. Possible explanations include nucleation in blade-wake vortices, heterogeneous condensation due to the effects of impurities in the steam and the effects of blade-row interaction unsteadiness. In $\S 4.2$, consideration is given to the third of these possibilities, namely the effect of the unsteadiness due to the interaction of blade-wakes with downstream blade rows. It will be shown that a direct result of these interactions is to dramatically broaden the droplet size distribution giving a general shape and mean diameter much more in keeping with experimental measurements in real turbines. Furthermore, the theory predicts that the formation of the liquid-phase takes place in an essentially unsteady manner and may encompass a region in the flow comprising one or more complete turbine stages in a multi-stage machine. The theory presents a radically different perspective of nucleation in turbines from the generally accepted view and, if correct, should have a major influence on the future development of calculation procedures for non-equilibrium steam flows in turbines.

\subsection{Effects of unsteadiness on the homogeneous nucleation of water droplets in multi- stage steam turbines}

The details of the theory are given by Guha \& Young (1994) and Guha (1995). The essence of the theory is that large-scale temperature fluctuations caused by the segmentation of blade wakes by successive blade rows have a dominating influence on nucleation and droplet growth in turbines. The fundamental premise is that, in passing through a multistage turbine, different fluid particles undergo different fluid mechanical experiences depending on the exact details of their passage through the machine and hence arrive at a given axial location with a wide variety of thermodynamic conditions. It is further assumed that, downstream of any turbine stage, the pressure of all the fluid particles would be nearuniform but their specific entropies (and hence static temperatures) would vary greatly depending on the dissipation experienced by a particular particle due to its being entrained in one or more blade boundary layers or lossy regions of the flow. However, although the path taken by a fluid particle is assumed to be random, the time-averaged dissipation of all the particles should agree with the overall loss distribution in the turbine. This is assumed known, either by direct measurement or from empirical loss correlations.

Figure 16 is a schematic diagram of the way in which the wakes from one blade row interact with, and are segmented by, the following row. It can be clearly seen that dissipation occurring in successive blade rows can become superposed in certain fluid particles (the darkly shaded areas).

A Lagrangian frame of reference is adopted and attention is focussed on a large number of individual fluid particles during their passage through the turbine. Homogeneous 


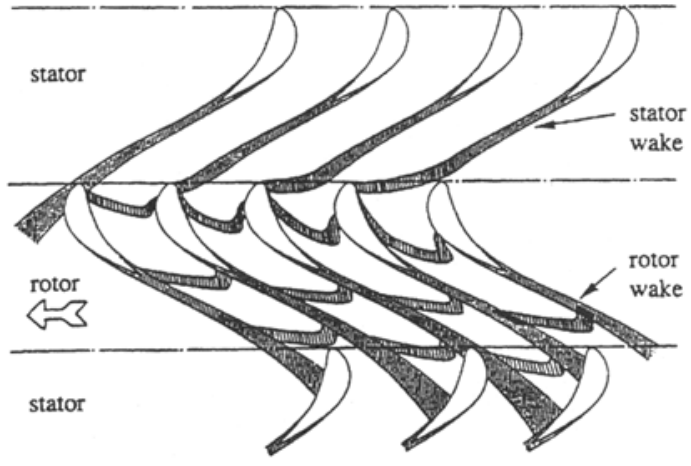

Figure 16. Schematic diagram of the passage of blade wakes through successive blade rows.

nucleation and growth of droplets in each fluid particle is assumed to be governed by classical theories. All fluid particles are assumed to experience the same pressure variation but those particles passing close to the blade surfaces suffer greater entropy production and therefore have higher static temperatures than those which pursue near-isentropic paths through the central portions of the blade passages. Particles which suffer high loss therefore nucleate later in the turbine than those which experience little dissipation. Condensation is thus viewed as an essentially random and unsteady phenomenon as the dissipation experienced by a fluid particle in one blade-row is assumed to be uncorrelated with its previous history. On a time-averaged basis, the condensation zone is spread over a much greater distance in the flow direction than a simple steady-flow analysis would indicate and may encompass several blade-rows depending on the number of stages in the machine. Predicted droplet size spectra show broad, highly-skewed distributions with large mean diameters and sometimes slight bimodality. These are all characteristics of experimentally measured spectra in real turbines. Conventional, steady-flow calculation methods, which predict a fixed Wilson point in a specific blade-row and a near-monodispersed droplet population, cannot reproduce any of these characteristics.

As shown in figure 17, a "loss profile" is constructed to represent the pitchwise distribution (from the suction to the pressure surface) of the loss in the blade-row. The pitchwise

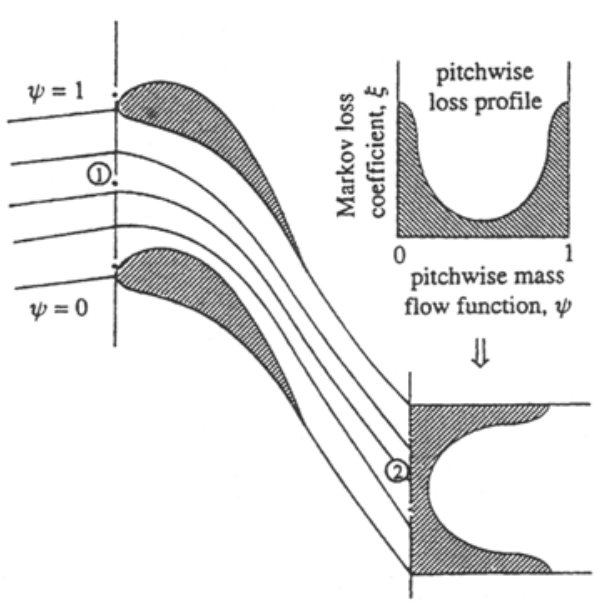

Figure 17. Specification of the pitchwise loss profile for a blade row. 
loss profiles represent the time-averaged entropy increase along particular pathlines but individual fluid particles associated with the passage of wakes may exit from the blade-row at different conditions because, on entry, their static temperatures and velocities deviate from the mean.

For the results presented below, a single (circumferentially averaged) pressure-time variation based on an axisymmetric streamline-curvature throughflow solution was adopted for all fluid particles. Fluid particles are then launched at the turbine inlet (where the steam is superheated), all at the same stagnation temperature and pressure. At the entry to each blade row a random number is generated that specifies the pathline to be followed by the particle. The pathline, in turn, fixes the value of the polytropic efficiency (figure 17). The "black-box" $(\$ 2.2)$ is then applied along the pathline to calculate the subcooling and the droplet size distribution (if nucleation has taken place) at the downstream of the particular blade row. A new random number is then generated that specifies the pathline in the next blade row and the procedure is repeated. The "black-box" can deal with successive nucleations after the primary as a matter of course should the expansion be sufficiently rapid to generate the high levels of subcooling required.

For each fluid particle, the subcooling and droplet size distribution at all points of interest are recorded. It is then a straightforward matter to compute the time-mean wetness fraction and other statistical properties in order to obtain a quantitative picture of the process of phase-change and liquid growth throughout the machine. In a six-stage lowpressure turbine, some $10^{4}$ fluid particle calculations are undertaken on each streamsurface to obtain converged statistical properties.

It has been assumed that the classical theories of homogeneous nucleation and droplet growth realistically describe the process of phase-change for individual fluid particles. In other words, if the pressure-time and temperature-time variations of a fluid particle during its passage through the turbine can be accurately specified, then it is assumed that the theories of nucleation and droplet growth correctly describe the rate of formation of the liquid-phase within that particle. For different fluid particles, phase-change will therefore be initiated at different locations in the machine, depending on the dissipation suffered by the particular particle. This is in contrast to the usual model of nucleation in turbines which assumes phase-change to be governed by the time-averaged fluid properties and therefore to occur at a single, well-defined location.

As an example calculation, the flow through the low pressure stages of a $320 \mathrm{MW}$ turbine was analysed. The turbine was manufactured by the Italian company "Ansaldo" and the complete geometry is available in the literature (Guha \& Young 1994; Guha 1995). The LP section has six-stages. Each stage consists of a stator followed by a rotor and hence there are twelve blade-rows altogether in the turbine.

The physical characteristics are best explained by adopting a Lagrangian viewpoint of a fluid particle as it passes through the turbine. As described in the previous sections, different fluid particles experience different amounts of dissipation and heat transfer, depending on the particular pathline followed. Two limiting cases can be identified. At one extreme are the fluid particles which always follow the mid-pitch pathline in each blade-row and consequently suffer no dissipation. They pursue an isentropic path to the Wilson point. At the other extreme are those particles which negotiate the regions of maximum loss in each 


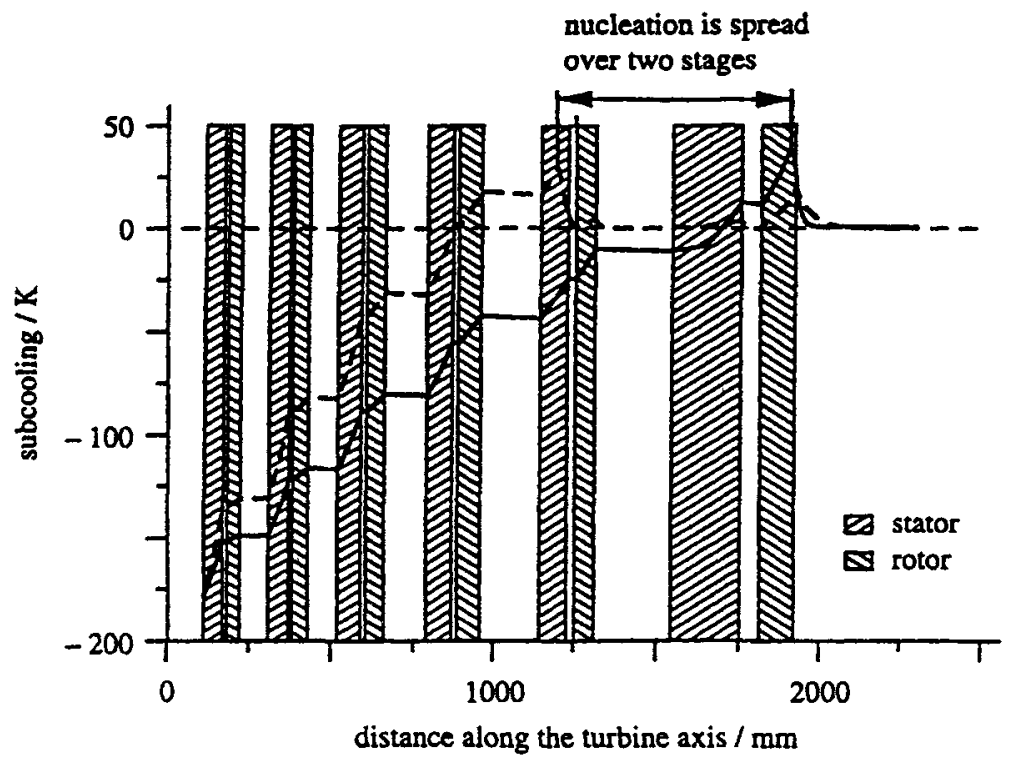

Figure 18. Axial variation in the Ansaldo turbine of vapour subcooling for two fluid particles representing the extreme cases of zero dissipation (- $\left.-{ }_{-}\right)$and maximum dissipation (-).

blade-row. Other fluid particles experience levels of loss intermediate between these two extremes.

Figure 18 shows the calculated variation of the vapour subcooling $\Delta T$ associated with the two extreme cases of zero and maximum dissipation. The subcooling of the fluid particles change as a result of the competing effects of the three physical processes: expansion, condensation and dissipation. Consider first the case of the fluid particle suffering no dissipation. Here, one of the mechanisms for altering the subcooling, i.e., dissipation, is absent. The fluid particle is superheated (negative $\Delta T$ ) at the turbine inlet. Its subcooling increases in each blade-row due to expansion but remains almost constant between the rows. It attains the Wilson point in the stator of the fifth stage and subsequently experiences an exponential decrease in $\Delta T$ due to the extremely rapid liberation of latent heat. $\Delta T$ increases significantly again in the last rotor where the expansion rate is too high to be offset by the counteracting effect of condensation. Much the same history is repeated for the fluid particle experiencing the maximum dissipation. Here, however, dissipation opposes the increase of the subcooling throughout the flow field. Consequently, the Wilson point occurs much further downstream (in the rotor of the last stage). Other fluid particles, experiencing intermediate amounts of dissipation, attain their Wilson points at intermediate locations between the two extremes. The region of nucleation thus covers (in a randomly unsteady manner) almost two complete turbine stages as opposed to being restricted to a very narrow zone in a specific blade-row.

A reliable "rule of thumb" is that Wilson points occurring at locations of higher expansion rate result in smaller droplets. The variable location of the Wilson point therefore results in large variations in mean droplet diameter. It should be understood, however, that the mean droplet diameters in the two limiting cases do not necessarily represent the 


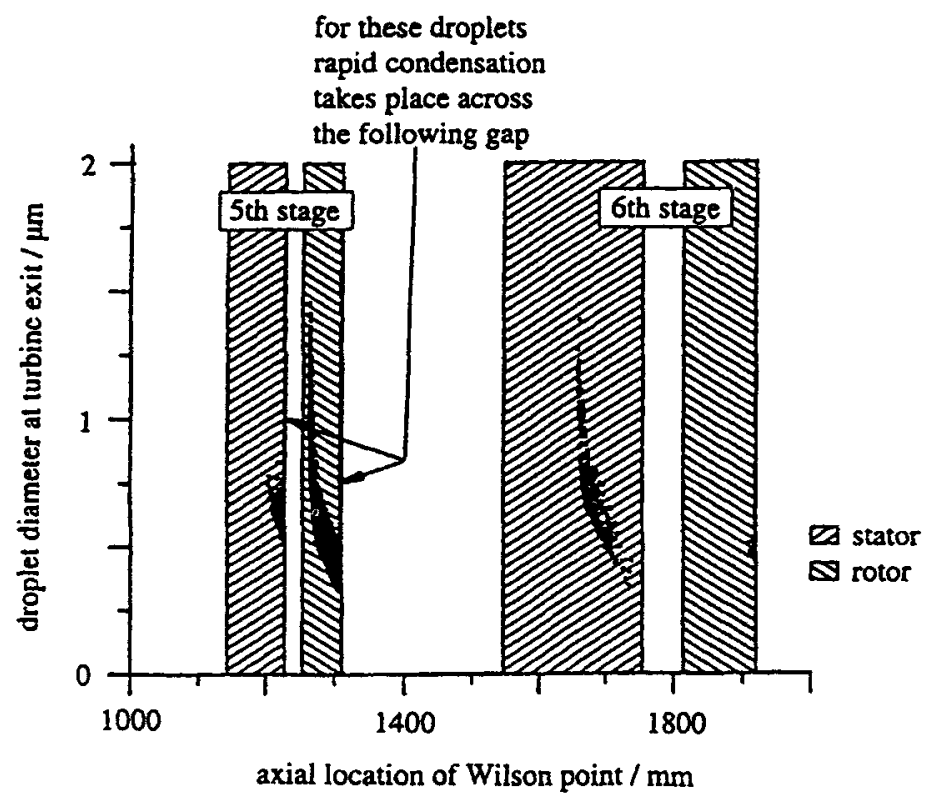

Figure 19. Computed Sauter mean droplet diameter at Ansaldo turbine outlet as a function of the axial location of the Wilson point for all fluid particles.

extreme limits of droplet size produced in the machine. The droplet size is dependent on the local expansion rate which does not vary monotonically with distance between the extreme locations of the Wilson points.

Figure 19 shows the location of the Wilson points for the totality of particles considered $\left(10^{4}\right)$. Each point on this diagram corresponds to an individual fluid particle. The abscissa denotes the axial location of the Wilson point and the ordinate denotes the mean diameter of droplets within the fluid particle on its arrival at the turbine outlet. (It should be appreciated that each fluid particle is actually composed of a polydispersed distribution of droplet sizes, details of which, although retained by the computational procedure, cannot be included in a single diagram such as figure 19.) It can be seen that the majority of fluid particles nucleate either in the fifth-stage rotor or in the sixth-stage stator. (The absence of Wilson points in the first part of the sixth-stage stator results from the very low expansion rate there, see (Guha \& Young 1994; Guha 1995).) In each blade-row, the mean diameter of the droplets becomes progressively smaller as the Wilson point moves towards the trailing-edge, as the rate of expansion tends to increase monotonically to each blade throat. However, interesting behaviour results for those fluid particles that reach the trailing-edge plane with subcoolings and nucleation rates which, although moderately high, are still insufficient to cause complete reversion to equilibrium. For these particles, the trailing-edge marks the Wilson point (i.e., the cessation of nucleation) even if comparatively few droplets have yet been produced. Reversion to equilibrium then occurs within the following gap by condensational growth on existing droplets. Because there is plenty of time available and because the droplet number density is low, these droplets may grow to very large sizes as shown in figure 19. Such fluid particles are then prime candidates for secondary nucleations in succeeding blade-rows because their liquid surface area is insufficient to 


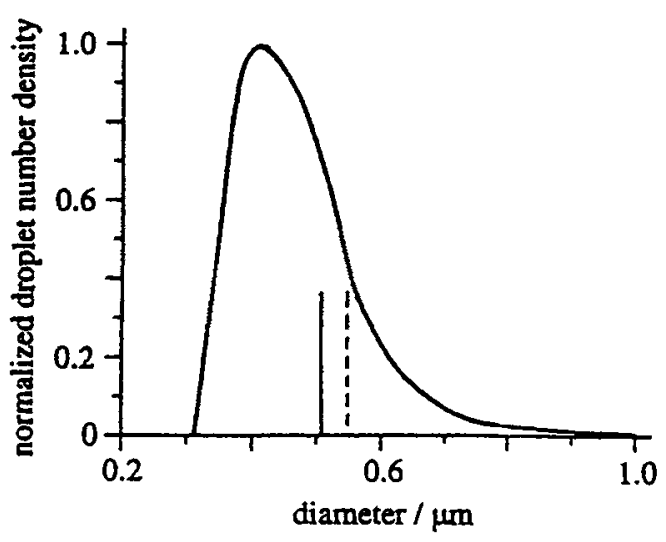

Figure 20. Computed time-averaged droplet size distribution at outlet of the Ansaldo turbine. - mass mean; - - Sauter mean.

offset (by condensation) the opposing effect of increased subcooling due to the rapid expansion.

An imaginary probe with unlimited resolution in space and time, sited at the turbine outlet, would register the complete droplet size distribution for each fluid particle it encounters. Real probes based on the measurement of attenuated or scattered light, however, record only sufficient information to deduce, at most, the time-averaged droplet size distribution (and sometimes only the time-averaged Sauter mean diameter). In order to compare the theoretical predictions with such measurements, a theoretical time-averaged droplet size distribution may be constructed at any axial location in the turbine if the diameters and number density of droplets in all the $10^{4}$ fluid particles considered are recorded by the computer for subsequent processing. The calculated time-averaged droplet size distribution for the Ansaldo turbine at outlet is shown in figure 20. The spectrum is polydispersed and highly-skewed (i.e., there is a large difference between the mean and most probable diameters) and resembles the shape of similar spectra measured in real turbines (figure 15). This is very significant, as no existing steady-flow calculation procedure can predict such a high degree of polydispersion.

Unfortunately, no measurement of the droplet size distribution is available for the Ansaldo turbine, although the time-averaged Sauter mean diameter of the droplets has been measured. The measured Sauter mean diameter at mid-span is about $0.4 \mu \mathrm{m}$, which is a little smaller than the calculated value of $0.55 \mu \mathrm{m}$, shown in figure 20 . However, allowing for the uncertainties and approximations in the calculation scheme, the level of agreement is extremely encouraging. Of course, many more experimental comparisons are required before it is possible to assert conclusively that the important physical processes are being successfully modelled by the theory presented.

In conclusion to this section, a theory has been developed for predicting the effect of temperature fluctuations on the homogeneous nucleation and growth of water droplets in multi-stage steam turbines. The fluctuations result from the segmentation of blade-wakes by successive blade-rows and the amplitude of the fluctuations increases with the number of stages. According to the model, the mechanics of nucleation in multi-stage turbines are quite different from the predictions of conventional steady-state theories of phase-change. For example, the nucleation zone may encompass (in a randomly unsteady manner) several blade-rows (as opposed to being isolated at a particular position in a specific blade-row). 
The inherent unsteadiness of the process also results in a highly-skewed, polydispersed (sometimes bimodal) time-averaged droplet size distribution, having similar characteristics to spectra measured in real turbines. The next step would be to include, in the calculation scheme, the effects of circumferential variation in pressure within the blade passages. More details may be found in Guha \& Young (1994) and Guha (1995).

The well-known books on multi-phase flows (e.g. by N A Fuchs and by G B Wallis) describe the long, inexhaustible list of multi-phase phenomena demonstrating their allpervasive occurrence. Here, we have considered only a few important topics, the selection inevitably being biased by the author's own interests: (See Guha $1997 \mathrm{~b}$ for a new theory for particle transport in turbulent flow.) We have used a complementary combination of analytical and computational techniques, and differential and integral treatments in order to model fundamental processes occurring in two-phase mixtures as well as to explain observed phenomena and experimental findings. This is an exciting, rewarding and potent field - so many interesting and important things remain to be done!

\section{References}

Guha A 1992a Jump conditions across normal shock waves in pure vapour-droplet flows. J. Fluid Mech. 241: 349-369

Guha A 1992 b Structure of partly dispersed normal shock waves in vapour-droplet flows. Phys. Fluids A 4: $1566-1578$

Guha A 1992c The physics of relaxation processes and of stationary and non-stationary shock waves in vapour-droplet flows. In Transport phenomena in heat and mass transfer (ed.) $\mathbf{J}$ Reizes (Amsterdam: Elsevier) pp 1404-1417

Guha A 1994a A unified theory of aerodynamic and condensation shock waves in vapour-droplet flows with or without a carrier gas. Phys. Fluids 6: 1893-1913

Guha A 1994b Thermal choking due to nonequilibrium condensation. Trans. ASME J. Fluids Engg. 116: 599-604

Guha A 1995 Two-phase flows with phase transition. VKI Lecture Series 1995-06 (von Karman Institute for Fluid Dynamics) pp 1-110

Guha A 1997a A unified theory for the interpretation of measured total pressure and total temperature in multiphase flows at subsonic and supersonic speeds. Proc. R. Soc. London 453: (in press)

Guha A 1997b A unified Eulerian theory of turbulent deposition to smooth and rough surfaces. J. Aerosol Sci. 28(8): (in press)

Guha A, Young J B 1989 Stationary and moving normal shock waves in wet steam. In Adiabatic waves in liquid-vapour systems (eds) G E A Meier, P A Thompson (Berlin: Springer) pp 159-170

Guha A, Young J B 1991 Time-marching prediction of unsteady condensation phenomena due to supercritical heat addition. Proc. Conf. Turbomachinery: Latest Developments in a Changing Scene (London: Institute of Mechanical Engineers) paper C423/057, pp 167-177

Guha A, Young J B 1994 The effect of flow unsteadiness on the homogeneous nucleation of water droplets in steam turbines. Philos. Trans. R. Soc. 349: 445-472

Young J B, Guha A 1991 Normal shock-wave structure in two-phase vapour-droplet flows. $J$. Fluid Mech. 228: 243-274 